\title{
Human Sebaceous Glands: How Studies of Skin Surface Lipids Have Provided Knowledge of Their Function and Control
}

\author{
John S. Strauss \\ University of lowa, lowa City, lowa, USA
}

Human sebaceous glands, especially of the face, have been the subject of intense study for many years, because of the importance of the glands in the pathogenesis of acne. Histological examination provides information on the structure of the sebaceous glands, but since there are great differences in sebaceous gland morphology from site to site in the glabrous skin of humans, there is great dependence on biopsy specimens taken from the face. Obtaining biopsy specimens from the face, especially multiple biopsies as necessitated in dynamic studies, has obvious limitations. There are other limitations that have to be taken into consideration. Of particular importance, there is a lack of uniformity in the size of the large sebaceous glands of the face. These sebaceous glands are found deep in the dermis.

Because of these limitations, a focus of many of the studies in our laboratories has been on the quantification of skin surface lipids and their composition. This has involved noninvasive procedures. It is important, however, to take into account that the skin surface lipids have a double component origin from both the sebaceous glands and the epidermis. Furthermore, for quantification, there is a necessity of isolating and trapping the skin surface lipids from a defined area.

Among the many questions that we have been able to answer are the following:
- What are the best ways to measure sebum composition and quantity?

- Which are the epidermal and which are the sebaceous components of skin surface lipids?

Of the major components of the surface film, triglycerides and cholesterol are of both epidermal and sebaceous origin. In contrast, wax esters and squalene are only derived from the sebaceous glands.

Can changes in sebaceous gland function with age be detected by the study of skin surface lipids? The changes from birth to old age will be detailed.

The study of skin surface lipids has helped define the androgenic dominance in the control of the sebaceous glands.

In addition, it has been possible to use measurements of sebum production to determine the status of certain endocrinological diseases involving the pituitary gland, the adrenal glands and the gonads.

In summary, the measurement and determination of the lipid components of skin surface lipid has indirectly provided us with the basic understanding of sebaceous gland function. While the complete understanding of sebaceous gland function is still to be determined, these studies have served as a foundation on which newer studies with cell and organ cultures using molecular biology are being pursued.

\section{KARGER \\ Fax +4161306 1234 \\ E-Mail karger@karger.ch}

www.karger.com
(C) 2006 S. Karger AG, Basel

$1018-8665 / 06 / 2133-0261 \$ 23.50 / 0$

Accessible online at:

www.karger.com/drm
Prof. John S. Strauss, MD

Professor Emeritus of Dermatology

University of Iowa

Iowa City, IA 52242 (USA)

Tel. +1 319351 6655, Fax +1 319356 6366, E-Mail jssstshaus@aol.com 